\title{
On the stability and mobility of di-vacancies in tungsten
}

\section{Heinola, K.}

2018-02

Heinola , K , Djurabekova , F \& Ahlgren , T 2018 , ' On the stability and mobility of

di-vacancies in tungsten ' , Nuclear Fusion , vol. 58 , no. 2 , 026004 . https://doi.org/10.1088/1741-4326/aa99ee

http://hdl.handle.net/10138/322672

https://doi.org/10.1088/1741-4326/aa99ee

acceptedVersion

Downloaded from Helda, University of Helsinki institutional repository.

This is an electronic reprint of the original article.

This reprint may differ from the original in pagination and typographic detail.

Please cite the original version. 


\title{
On the stability and mobility of di-vacancies in tungsten
}

\author{
K. Heinola ${ }^{1}{ }^{*}$ F. Djurabekova ${ }^{1,2}$, and T. Ahlgren ${ }^{1}$ \\ EUROfusion Consortium \\ ${ }^{1}$ Department of Physics, \\ University of Helsinki, P.O. Box 64, \\ 00560 Helsinki, Finland \\ ${ }^{2}$ National Research Nuclear University MEPhI, \\ Kashirskoye sh. 31, 115409 Moscow, Russia
}

\begin{abstract}
Properties of small vacancy clusters in tungsten were studied with first-principles calculations. The binding and formation energies of the vacancy clusters increase with the cluster size. Dynamic characteristics of the di-vacancy was specified between room temperature and $700 \mathrm{~K}$ with lattice kinetic Monte Carlo calculations, which were parametrised with the present firstprinciples results for the dissociation barriers. An Arrhenius fit for the di-vacancy diffusion yielded $D=0.04 \exp (-1.65 \mathrm{eV} / k T) \mathrm{cm}^{2} \mathrm{~s}^{-1}$ and for the mean lifetime $\tau=0.093 \exp (1.7 \mathrm{eV}) / k T$ ps. The di-vacancy system was found to be stable up to $500 \mathrm{~K}$ due to its high energy needed for dissociation. Having a carbon impurity was found to increase the tungsten di-vacancy binding energy.
\end{abstract}

\footnotetext{
* Corresponding author. E-mail: kalle.heinola@helsinki.fi
} 


\section{INTRODUCTION}

Irradiation-induced defects in solids is an important study subject in the field of materials science [1]. The formation of large-scale defects, such as voids, cavities and even blistering of the material, is always initiated by the evolution of the immediate primary damage, i.e. the point defects, in the irradiated material. Therefore, understanding of the formation and kinetics of these primary microscopic defects is of fundamental importance in order to control the observed final defect production. This is considered to be vital especially with materials which are placed to withstand heavy irradiation conditions. In the present work, the studied material is tungsten (W), which has been chosen as a first-wall (FW) material in the incomparable irradiation conditions of the thermonuclear fusion reactor [24]. The FW is subjected to abrupt high energy plasma particle bursts during plasma Edge Localised Modes (ELMs). Moreover, a fraction of the irradiation exposure is due to high energy tritium $(\mathrm{T})$ ions from the fusion reactions in the deuterium plasma (D-D). However, with D-T plasmas, in addition to the ELMs, the high flux of energetic $14 \mathrm{MeV}$ neutrons from the fusion reactions increase substantially the radiation damage in the FW materials. These ion and neutron-induced damage worsen the material properties, such as, lowering the temperature required for ductile-to-brittle phase change. Moreover, the damaged regions within the FW can result as acting as sinks for the unwanted trapping of fusion fuel T, which in turn is a radiological hazard in the next-step fusion devices, such as ITER [5].

In the course of irradiation, the ionic and neutron projectiles after penetrating the surface of the target material will interact with the host matrix atoms. The range of these interactions vary depending on the projectile type - neutron-induced damage extends evenly deep in the FW material, whereas the ion-induced damage creation is affected by slowing down of the ion due to nuclear and electronic stopping power making the resulted damage narrower and closer to the FW surface. Depending on the energetics of these interactions due to ions or neutrons, primary damage will be formed in the form of Frenkel pairs in which the host atoms are kicked out of their lattice sites leaving a vacant site behind. The number of stable Frenkel pairs created depend mainly on the energy of the incoming particle and the displacement energy of the target material. The vacant sites can form clusters already during the displacement events or cascades of displacements. The present study focuses on the stability of the small vacancy clusters in body-centered cubical (bcc) W. 
TABLE I. The DFT calculated formation $\left(E_{f}\right)$ and binding $\left(E_{b}\right)$ energies for $\mathrm{V}_{n}$ systems in W with $1 \leq n \leq 4$. Results are compared with experimental and other DFT results. Units eV.

Expt.

\section{DFT}

Other

Present work

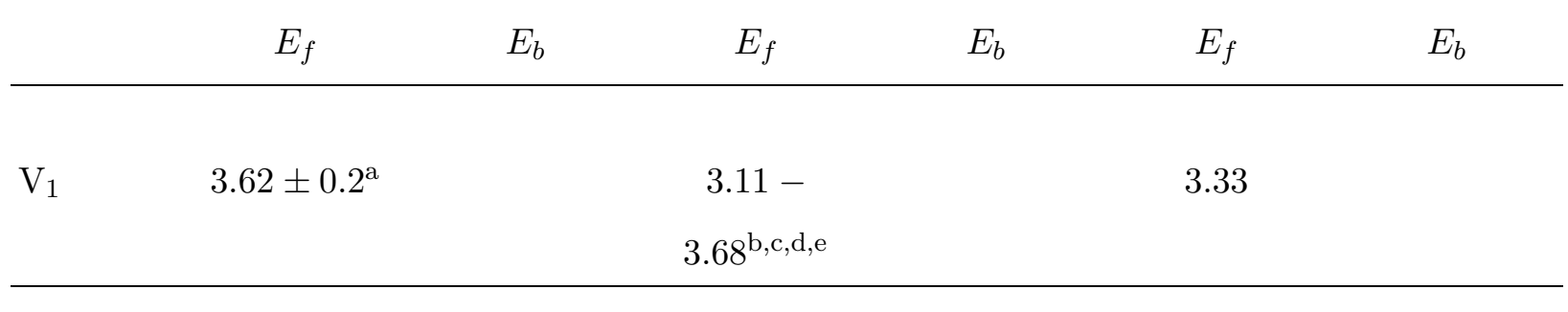

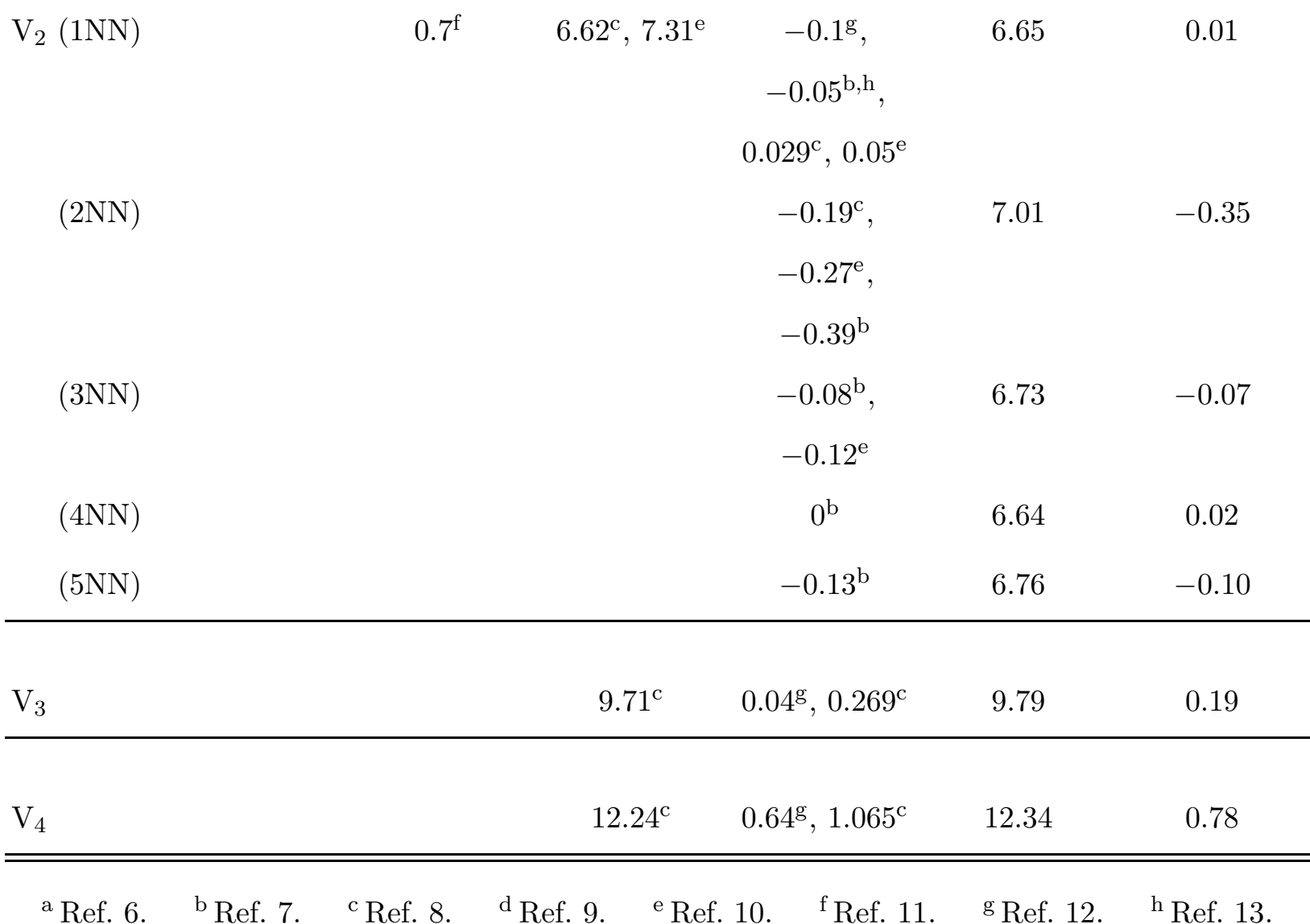

${ }^{\mathrm{a}}$ Ref. 6. $\quad{ }^{\mathrm{b}}$ Ref. $7 . \quad{ }^{\mathrm{c}}$ Ref. 8. $\quad{ }^{\mathrm{d}}$ Ref. 9. $\quad{ }^{\mathrm{e}}$ Ref. 10. $\quad{ }^{\mathrm{f}}$ Ref. $11 . \quad{ }^{\mathrm{g}}$ Ref. $12 . \quad{ }^{\mathrm{h}}$ Ref. 13.

The primary vacancy clustering phenomenon in $\mathrm{W}$ can still be considered as an unanswered question. The vacancy clustering with different cluster sizes have been observed experimentally $[14,15]$, but the theoretical explanation is missing. According to recent 
theoretical first-principles calculations with electron density functional theory (DFT) the initial stage of a vacancy cluster, i.e. the di-vacancy, is unstable or barely stable in pure W $[7,13]$ leading to speculative conclusions to the formation of larger vacancy clusters. One of the many explanations for making the $\mathrm{W}$ di-vacancy stable, and thus allowing formation of larger clusters, is the presence of impurities in the $\mathrm{W}$ samples.

In the present research, the stability of small vacancy clusters in $\mathrm{W}$ was studied computationally. The main study subjects were the stability and dissociation dynamics of the di-vacancy in pure bulk W, which were scrutinised with DFT and Lattice Kinetics Monte Carlo (LKMC) calculations, respectively. As a part of the present work, DFT was used for studying the effect of carbon $(\mathrm{C})$ impurity to the di-vacancy stability, since $\mathrm{C}$ is typically the most abundant non-metallic intrinsic impurity found in $\mathrm{W}(\lesssim 10 \mu \mathrm{g} / \mathrm{g}$ in high-purity 99.99\% poly-crystalline W by Plansee AG).

DFT calculations were performed for determining the energetics of vacancies and the dissociation barriers of a di-vacancy. The obtained barrier heights for pure $\mathrm{W}$ were further used in parametrising the dynamical calculations performed with LKMC method at temperatures between RT and $700 \mathrm{~K}$. The presented LKMC and DFT results show the temperature dependence of the di-vacancy dissociation and the stabilising effect of the carbon impurity.

\section{COMPUTATIONAL DETAILS}

\section{A. Electron density functional theory}

The DFT calculations were performed for determining the ground state energies of the studied W vacancy systems. The Vienna Ab-initio Simulation Package (VASP) [16-18] was chosen for this purpose. The electronic ground-state was calculated using the projectoraugmented wave (PAW) potentials [19, 20] as provided in VASP. For the volumetric and ionic relaxation the conjugate gradient algorithm was used and the plane wave energy cutoff was $450 \mathrm{eV}$. The large energy cut-off was used in order to reach high accuracy with every PAW potential used for the elements in this work. No symmetrizations were applied. The electronic and ionic relaxation cut-offs for high-accuracy calculations were chosen as $10^{-5} \mathrm{eV}$ and $10^{-3} \mathrm{eV} / \AA$, respectively. The electron exchange-correlation was described with generalised gradient approximation using Perdew-Burke-Ernzerhof functionals [21, 22]. The 
six outermost electrons ( $5 d$ and $6 s$ sub-shells) of the $\mathrm{W}$ atom were used as valence electrons. For the impurity atom $\mathrm{C}$ the four $(2 s$ and $2 p)$ outermost electrons were kept in the valence. The partial occupancies were integrated with the Methfessel and Paxton method [23] of the first order. A $3 \times 3 \times 3 k$-point mesh was sampled with Monkhorst and Pack scheme [24]. The convergence obtained with DFT parameters was validated and further checked to reproduce the experimentally obtained properties of $\mathrm{W}[25,26]$. Supercell with 128 lattice cites was used in the calculations. The present studied systems were mono- $\left(\mathrm{V}_{1}\right)$, di- $\left(\mathrm{V}_{2}\right)$, tri- $\left(\mathrm{V}_{3}\right)$ and four-vacancy $\left(\mathrm{V}_{4}\right)$. The $\mathrm{V}_{3}$ and $\mathrm{V}_{4}$ were triangular structures as found in Refs. 8 and 27. From the relaxed ground states the corresponding formation and binding energies were determined.

For the di-vacancy, once the geometry with the lowest energy state was found, the divacancy dissociation energetics up to five nearest-neighbour (NN) configurations was determined with the nudged elastic band (NEB) method [28, 29]. The NN positions are presented in Fig. 1. Seven intermediate images with two endpoint images were used in NEB for finding the geometries giving the minimum energy for the di-vacancy dissociation paths.

Finally, the ground state energies and the resulted dissociation barrier height calculations have been performed by finding their fully relaxed ground states at $0 \mathrm{~K}$. Hence the DFT calculations in this work do not take into account the contribution of the change in the atomistic vibrational entropies $\left(-T \Delta S_{\text {vib }}\right)$ when compared a defect-free system to a system with vacancies. However, the $\Delta S_{\text {vib }}$ can be assessed to be small yielding to a significant contribution only at high $T$. In Sect. IB is shown the need to limit the LKMC calculations to low temperatures from RT to $700 \mathrm{~K}$ providing the change in free energy due to $-T \Delta S_{\text {vib }}$ to be minuscule.

\section{B. Lattice kinetic Monte Carlo}

LKMC technique has proven to be an efficient tool in estimating ageing of materials over many years with respect to the thermally activated evolution of extended defects (such as nucleation, growth, annihilation etc.), provided that all the activation barriers for the corresponding transitions are available [30-34]. In the present work, the atomistic LKMC approach has been applied in investigating the behaviour of a single di-vacancy cluster in tungsten at different temperatures, assuming the two vacancies being able to come close 
to one another via random walk mechanism in a bcc lattice structure. Strictly speaking the probability of formation of a di-vacancy depends on the concentration of vacancies in tungsten and the diffusion coefficient of a mono-vacancy, but presently we aim to estimate the lifetime and the mobility of a di-vacancy after it was formed. For this study the LAKIMOCA code [35] was used, which employs the residence time algorithm [36] to assess the migration kinetics of a small cluster within its lifetime. The LAKIMOCA algorithm with the calculation of transition barriers explicitly bound to the initial and final states, however, was modified similarly to the earlier work in Ref. 34 .

It has been previously shown that the activation barriers rigorously calculated with the account for relaxation effects in the structure, may lead to new insight on the kinematics of the process, which otherwise escapes the sight of a researcher if the relaxation is neglected [32]. The relaxation effect can be obtained either by Molecular Dynamics simulations applying the appropriate empirical potentials, or by more advanced DFT calculations. For the present study, DFT calculations were used to determine the transition barriers between different states of a di-vacancy as described in Sect. I A. Each state of the di-vacancy was distinguished by the distance between the two vacancies. These were considered to form a cluster while the distance between the vacancies was ranging from first nearest neighbour $(1 \mathrm{NN})$ distance to $5 \mathrm{NN}$ one. Vacancies separated by more than a distance of $5 \mathrm{NN}$ were considered to represent a dissociated di-vacancy.

In the LKMC simulations the tungsten sample was constructed as $20 \times 20 \times 20$ unit cells in the bcc structure, comprising two vacancies. In the LKMC calculations the probability of di-vacancy formation was not considered. For this reason the actual size of the cell was not of importance: two vacancies were considered be formed as a di-vacancy cluster until they were recorded to be split, i.e. moved apart at more than 5NN distance; no re-joining events were taken into consideration. The first simulation was performed at RT and the temperature was increased with an interval of $100 \mathrm{~K}$. The statistics was collected over 200 Monte Carlo trials for each temperature. At $700 \mathrm{~K}$ the di-vacancy lifetime became vanishing small $(\sim 100 \mathrm{~ms})$ and the LKMC were discontinued to higher temperatures. 


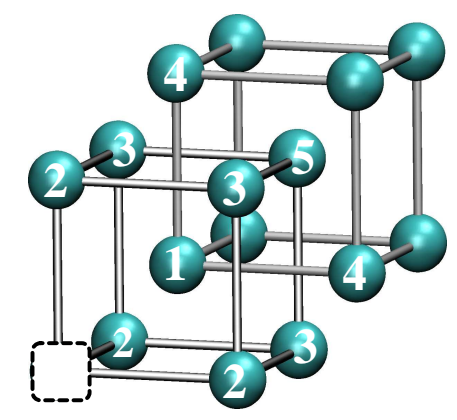

FIG. 1. The bcc di-vacancy nearest neighbour configurations used in the DFT and lattice KMC calculations. The five nearest neighbour configurations are numbered with respect to the monovacancy denoted with a dotted square.

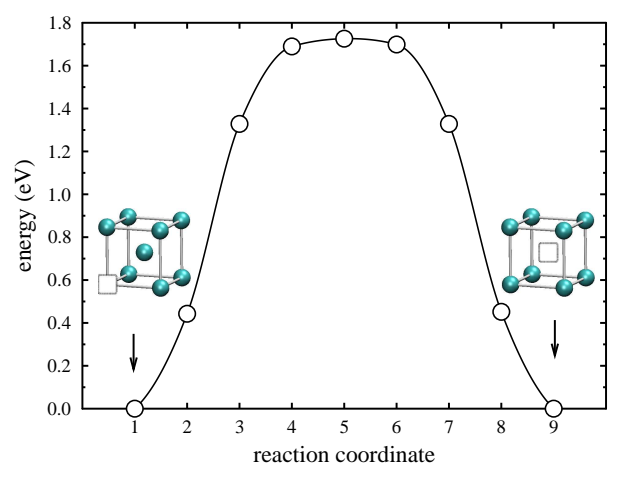

FIG. 2. The W mono-vacancy migration barrier as obtained with NEB-DFT.

\section{RESULTS}

\section{A. Di-vacancy energetics with DFT}

For validating our DFT results the properties of bulk W have been calculated and compared with experiments and other DFT calculations found in the literature. The examined bulk W lattice structures were the bcc, fcc and A15 geometries. The results have been published elsewhere [25]. The DFT calculated properties for $\mathrm{W}$ mono-vacancy have been presented previously [27, 37]. Our results for the vacancy formation and migration energies agree with the experimental results [6] and with other DFT calculations [9]. In addition, the DFT parameters used have reproduced the reconstruction of the $\mathrm{W}(100)$ surface [26].

The incremental binding energy $E_{b}$ of a point defect cluster containing $2 \leq n \leq 4$ defects 

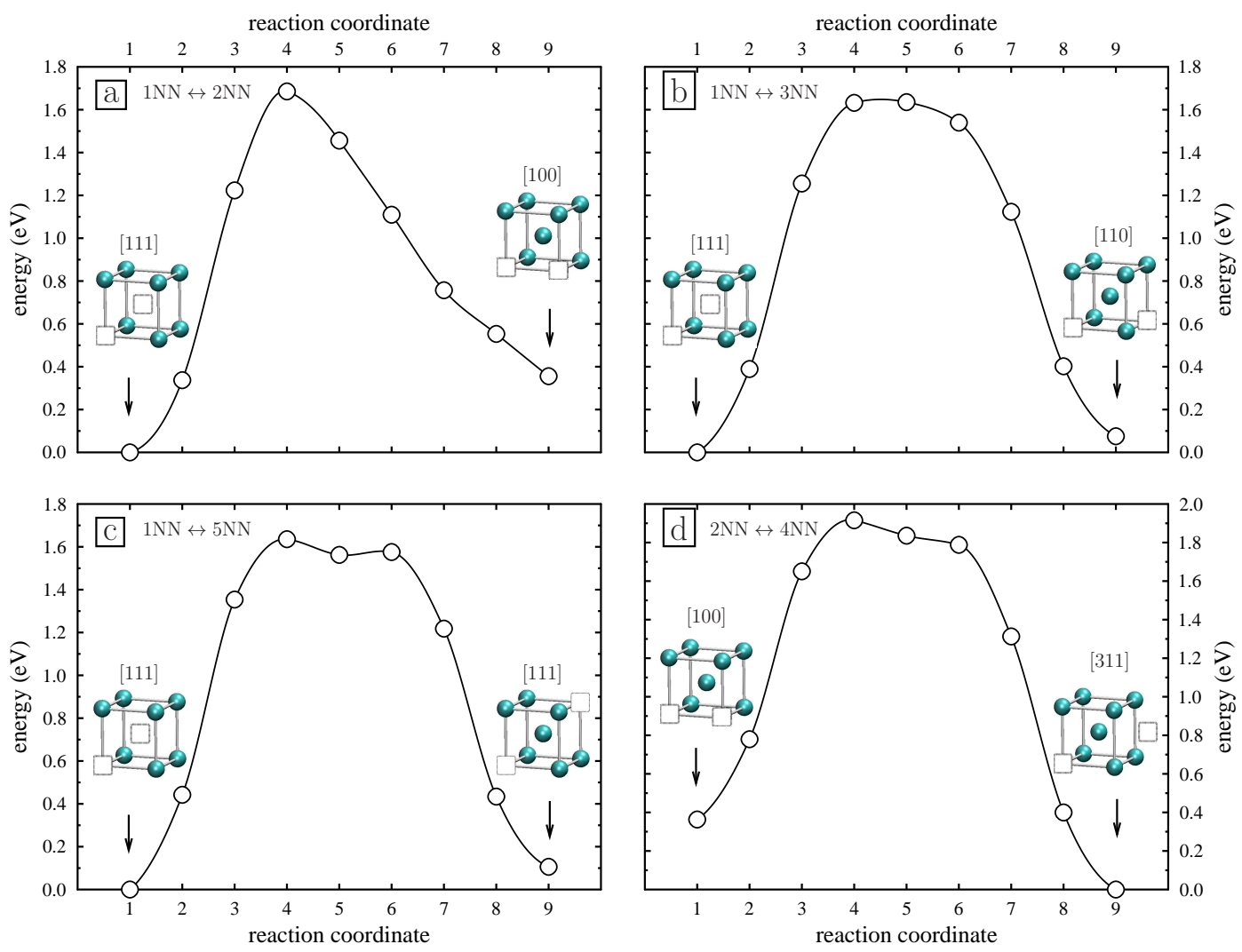

FIG. 3. (Color online) The W [111] (1NN) di-vacancy transition barriers to the neighbouring states a) [100] (2NN), b) [110] (3NN) and c) extended [111] (5NN) as obtained with NEB. Also shown d) the barrier between [100] $(2 \mathrm{NN})$ and [311] $(4 \mathrm{NN})$. Corresponding unit cell geometries of the end points are visualised.
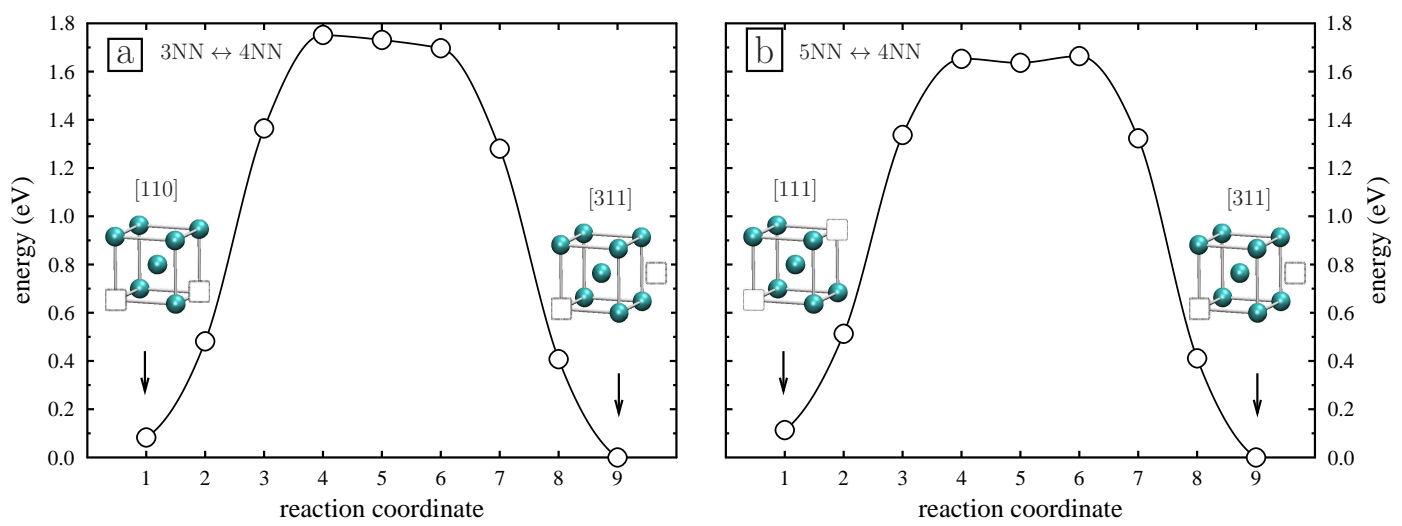

FIG. 4. (Color online) The transition barriers of a) [110] (3NN) and b) extended [111] (5NN) divacancy to the [311] (4NN) orientation as obtained with NEB. Corresponding unit cell geometries of the end points are shown. 
was calculated with

$$
E_{b}(n)=[E(n-1)+E(1)]-\left[E(n)+E_{0}\right] .
$$

$E(n)$ is the total energy of the studied defect system, and $E(n-1)$ is the total energy of one defect dissociated from it. $E(1)$ is the energy of the host lattice with one point defect and $E_{0}$ is the reference energy of perfect bcc W. If the binding energy calculated with Eq. (1) is positive, the defect system is more stable than the system with one defect separated from it. The energy needed for a particle to be completely freed from the cluster, i.e. the energy needed for detrapping $E_{t}$, is usually defined as the binding energy summed with the migration energy of the fastest particle

$$
E_{t}=E_{b}+E_{m}
$$

The migration barrier for $\mathrm{W}$ mono-vacancy $\left(E_{m}^{\mathrm{V}}=1.7 \mathrm{eV}\right)$ has been presented in our previous work $[27,37]$.

The results of the binding energies $E_{b}$ for the $\mathrm{V}_{n}$ clusters with $2 \leq n \leq 4$ are presented and compared to other works in Table I. The $E_{b}$ values agree well with the other theoretical values obtained with DFT [38].

The energetics of the $\mathrm{W}$ di-vacancy is an intriguing question. If two mono-vacancies do not form a di-vacancy system thermodynamically, the agglomeration of vacancies into clusters in pure bulk W would be only possible with large mono-vacancy concentrations where the formation of a tri-vacancy, i.e. simultaneous assimilation of three mono-vacancies, will increase its probability. The only experimental $E_{b}$ value for the $\mathrm{W}$ di-vacancy is $0.7 \mathrm{eV}$ [11]. In an early DFT study it was stated that the $\mathrm{W}$ di-vacancy is unstable even as the two vacancies are separated as far as in the $5 \mathrm{NN}$ position [7]. The results showed the $2 \mathrm{NN}$ geometry being the least favorable configuration with the highest negative $E_{b}$. Interestingly however, the lowest negative $E_{b}$ was found for the $4 \mathrm{NN}$ geometry, and was shown to be nearly null in that work. Numerical values extracted from Ref. 7 are shown in Table I. A similar $E_{b}$ trend for $1 \mathrm{NN}$ to $5 \mathrm{NN}$ was obtained later by Ventelon et al. [13] who found the $2 \mathrm{NN}$ being the least favourable configuration with $E_{b}=-0.46 \mathrm{eV}$, and the $4 \mathrm{NN}$ geometry was shown to have clearly the lowest negative $E_{b}$. DFT work by Kato et al. [10] yielded $E_{b}$ values of $0.05,-0.27$ and $-0.12 \mathrm{eV}$ for the $1^{\text {st }}, 2^{\text {nd }}$ and $3^{\text {rd }} \mathrm{NN}$ di-vacancies, respectively. That work reported the di-vacancy $E_{b}$ having a positive value at $1 \mathrm{NN}$, which would allow it to be a thermodynamically stable entity. However, no $E_{b}$ value for $4 \mathrm{NN}$ geometry was 
reported. More recent DFT results show similar $E_{b}$ energetics for $1 \mathrm{NN}$ and $2 \mathrm{NN}$ geometries with $0.007 \mathrm{eV}$ and $-0.190 \mathrm{eV}$ [8]. Our result follow the same trend for $1^{\text {st }}$ to $3^{\text {rd }} \mathrm{NN}$ divacancies with the corresponding $E_{b}$ values of $0.01,-0.35,-0.07 \mathrm{eV}$. Further, our $E_{b}$ results for $4 \mathrm{NN}$ and $5 \mathrm{NN}$ were found to be 0.02 and $-0.10 \mathrm{eV}$, respectively. Based on this the most stable di-vacancy configurations in bcc W would be the $1 \mathrm{NN}$ and $4 \mathrm{NN}$ geometries. The di-vacancy is found to be a semi-stable state in the sense that albeit its $E_{b}$ for $1 \mathrm{NN}$ or $4 \mathrm{NN}$ is minuscule, it still can be present as a stable system at a certain temperature range, since the dissociation barrier, i.e. the energy required for detrapping, hinders either of the vacancies to be apart from each other. This feature has been also observed and studied recently by Oda et al. [39] by using DFT and NEB calculations. From the kinetics point of view, as the temperature increases the effect of the barrier acting as a dissociation limiting step diminishes and the over-barrier motion becomes thermodynamically more probable.

In Ref. 40 it was demonstrated with first-principles calculations, that the variations in the bimodal shape of the density of the electronic states (DOS) at the Fermi level $\left(E_{\mathrm{F}}\right)$ play a crucial role in the $\mathrm{V}_{1}$ properties of bcc transition metals. The position of the $E_{\mathrm{F}}$ in the DOS pseudogap contributes to $E_{f}$ and $E_{m}$, and has an effect to the electronic contribution on formation and migration entropies. For the $\mathrm{V}_{2}^{1 \mathrm{NN}}$ and $\mathrm{V}_{2}^{2 \mathrm{NN}}$ defect geometries in $\mathrm{W}$ it has been shown the $\mathrm{V}_{2}^{2 \mathrm{NN}}$ giving an increase in the $d$-band local DOS at $E_{\mathrm{F}}$ pseudogap as compared to the corresponding $\mathrm{V}_{2}^{1 \mathrm{NN}}$ pseudogap [13]. This result is confirmed in the present work, and the shift in the $d$-band may be attributed to the difference in the geometrical structures of $\mathrm{V}_{2}^{1 \mathrm{NN}}$ and $\mathrm{V}_{2}^{2 \mathrm{NN}}$ as follows. The empty lattice site $\mathrm{V}_{1}$ in bcc $\mathrm{W}$ affects the electronic structure of its eight nearest-neighbour atoms, which is seen as variation in their local $d$-bands. The $\mathrm{V}_{2}^{2 \mathrm{NN}}$ structure influences to its twelve nearest-neighbour $\mathrm{W}$ atoms in the [100] direction. Four out of these twelve W atoms reside on the centre plane (010) of the $\mathrm{V}_{2}^{2 \mathrm{NN}}$ geometry, and are joint nearest-neighbour atoms of the two vacancies. As a result, these shared four $\mathrm{W}$ atoms are influenced by additional dangling bonds giving rise to the electronic groundstate of the system. The $\mathrm{V}_{2}^{1 \mathrm{NN}}$ geometry influences its ten nearestneighbour $\mathrm{W}$ atoms of which none is shared by the two vacancies. In this case the electronic system is less distorted (less variance in the local DOS) giving a lower electronic groundstate energy. Strong repulsion of $\mathrm{V}_{2}^{2 \mathrm{NN}}$ in $\mathrm{W}$ is a particular feature among bcc transition metals [13] and further investigations are needed for scrutinizing the effect of local DOS variation to the stability of the di-vacancies in bcc transition metals. The $\mathrm{V}_{2}^{3 \mathrm{NN}}$ structure has sixteen $\mathrm{W}$ 


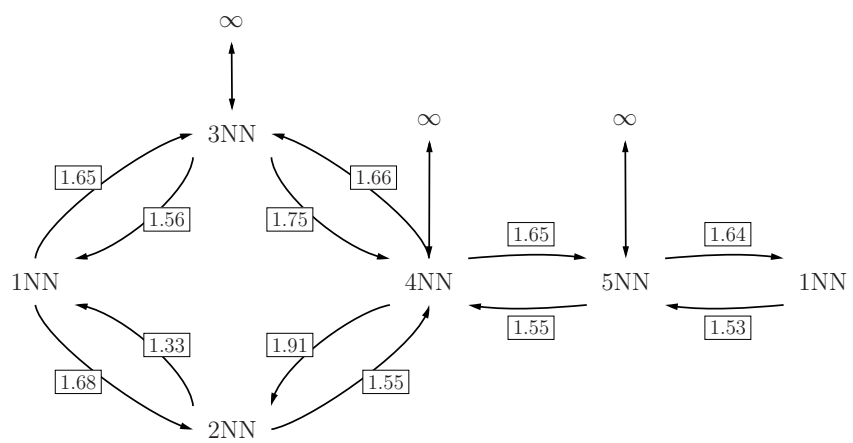

FIG. 5. DFT obtained potential barrier heights (boxed values in units of eV) used in the KMC calculations for di-vacancy NN transitions. Barrier to split the di-vacancy, i.e. transition from $3 \mathrm{NN}, 4 \mathrm{NN}$ and $5 \mathrm{NN}$ to $\infty$, was taken as $1.7 \mathrm{eV}$.

nearest-neighbours of which two are shared with the vacancies. As a final note, for $\mathrm{V}_{2}^{4 \mathrm{NN}}$ and $\mathrm{V}_{2}^{5 \mathrm{NN}}$ the number of $\mathrm{W}$ nearest-neighbours is sixteen for both structures, but the number of shared W nearest-neighbours is zero and one, respectively. There appears to be a correlation with the above-mentioned number of shared $\mathrm{W}$ atoms for various $\mathrm{V}_{2}$ geometries and their corresponding $E_{b}$ values (Table I). Confirming this trend with respect to the effect of local DOS variation in $\mathrm{W}$ and other bcc transition metals will be part of a future work.

In Fig. 5 are presented the possible di-vacancy dissociation energetics from $1 \mathrm{NN}$ and from $4 \mathrm{NN}$ geometries. Once a $1 \mathrm{NN}$ geometry is formed, the allowed di-vacancy dissociation pathways found are to $2 \mathrm{NN}$ and to $3 \mathrm{NN}$ geometries. The formed $4 \mathrm{NN}$ structure can dissociate to $2 \mathrm{NN}, 3 \mathrm{NN}$ and $5 \mathrm{NN}$ geometries. In Fig. 5 reported energies for over-barrier motion suggest the $1 \mathrm{NN}$ being a more attractive di-vacancy configuration than the $4 \mathrm{NN}$, since it is energetically more favorable for a mono-vacancy to go back and get trapped to a $1 \mathrm{NN}$ than to a $4 \mathrm{NN}$ geometry. This result is confirmed with LKMC calculations presented in Sect. II B and in Fig. 7.

Recent work on formation of $\mathrm{W}$ di-vacancies have shown that the di-vacancies can be stabilised by enriched hydrogen [10]. In that work the most stable configuration was the $2 \mathrm{NN}$ di-vacancy with hydrogen $\mathrm{V}_{2}^{2 \mathrm{NN}} \mathrm{H}_{1}$. Similar di-vacancy stabilisation effect can be expected with enriched helium due to its ability to bind strongly with the $\mathrm{W}$ mono-vacancy $[7,41]$. In the present work, the effect of carbon (C) impurity atom was examined. It was found, in the presence of $\mathrm{C}$ impurity the $2 \mathrm{NN}$ di-vacancy becomes more stable than the $1 \mathrm{NN}$ divacancy: the corresponding $E_{b}$ for $\mathrm{V}_{2}^{2 \mathrm{NN}} \mathrm{C}_{1}$ and $\mathrm{V}_{2}^{1 \mathrm{NN}} \mathrm{C}_{1}$ is $1.29 \mathrm{eV}$ and $0.04 \mathrm{eV}$ (see Table I). 
The stabilization of the $\mathrm{V}_{2}^{2 \mathrm{NN}}$ is due to the $\mathrm{C}$ residing in-between the two vacancies at an octahedral site bonding with the four nearest-neighbour W atoms. These four nearestneighbour $\mathrm{W}$ atoms in the (010) plane have two dangling bonds each due to the missing $\mathrm{W}$ atoms forming the $\mathrm{V}_{2}^{2 \mathrm{NN}}$ configuration in the [100] direction. The electronic groundstate of this system gets stabilized by the add-electrons provided by the $\mathrm{C}$ impurity atom located at the abovementioned octahedral site: this is the nearest position with respect to the four $\mathrm{W}$ atoms having two dangling bonds, and the $\mathrm{C}$ atom shares its valence electrons thus forming a bond with these host lattice atoms. In the $\mathrm{V}_{2}^{1 \mathrm{NN}}$ configuration the surrounding W atoms have only single dangling bonds, and the most stable location for a $\mathrm{C}$ impurity was found on the octahedral site in one of the mono-vacancies in the $\mathrm{V}_{2}^{1 \mathrm{NN}}$ system. Thus, the $\mathrm{C}$ atom can be considered interacting only with the $\mathrm{V}_{1}$ it is residing in, whereas in the $\mathrm{V}_{2}^{2 \mathrm{NN}}$ configuration the $\mathrm{C}$ atom interacts with the whole system via residing in-between the two $\mathrm{V}_{1}$ 's. Interestingly, the $\mathrm{DFT} E_{b}$ result for the $\mathrm{V}_{2}^{1 \mathrm{NN}} \mathrm{C}_{1}$ configuration suggests, that it is thermodynamically nearly equal configuration as the $\mathrm{V}_{1} \mathrm{C}_{1}$ system with a $\mathrm{V}_{1}$ far from it (Table II). Further, the $E_{b}$ for $\mathrm{V}_{2}^{2 \mathrm{NN}} \mathrm{C}_{1}$ obtained with DFT indicate (Table II), that at high temperatures the $2 \mathrm{NN} \mathrm{V}_{2} \mathrm{C}_{1}$ could dissociate as $\mathrm{V}_{2}^{2 \mathrm{NN}} \mathrm{C}_{1} \rightarrow \mathrm{V}_{1} \mathrm{C}_{1}+\mathrm{V}_{1}$. Taking into account the $\mathrm{V}_{1}$ migration energy $E_{m}^{\mathrm{V}_{1}}=1.7 \mathrm{eV}$ results into detrapping energy of $\sim 3 \mathrm{eV}$. This leads to the conclusion that the $\mathrm{C}$-di-vacancy system $\mathrm{V}_{2}^{2 \mathrm{NN}} \mathrm{C}_{1}$ is kinetically stable up to extremely high temperatures.

\section{B. Kinematics of $\mathrm{W}$ di-vacancy}

As described in Sect. IB, the stability of the di-vacancy was determined with lattice KMC method using the LAKIMOCA [42]. The mono-vacancy diffuses via jumps to nearestneighbouring lattice cites in the [111] direction. Thus, a limited number of available cites are for a vacancy to jump into and from the di-vacancy system. Longer jumps in the [100] direction result into large potential barrier which hinders the vacancy motion. For example, a NEB calculation with DFT for $1 \mathrm{NN} \leftrightarrow 4 \mathrm{NN}$ transition resulted to a potential barrier of $5 \mathrm{eV}$, which leads to a energetically highly improbable transition.

The residence time algorithm implemented in the LAKIMOCA code allows to investigate the kinematics of the system evolution, since it assesses the time, which the system spends for each transition. Considering the entire population of the non-interacting di-vacancies 


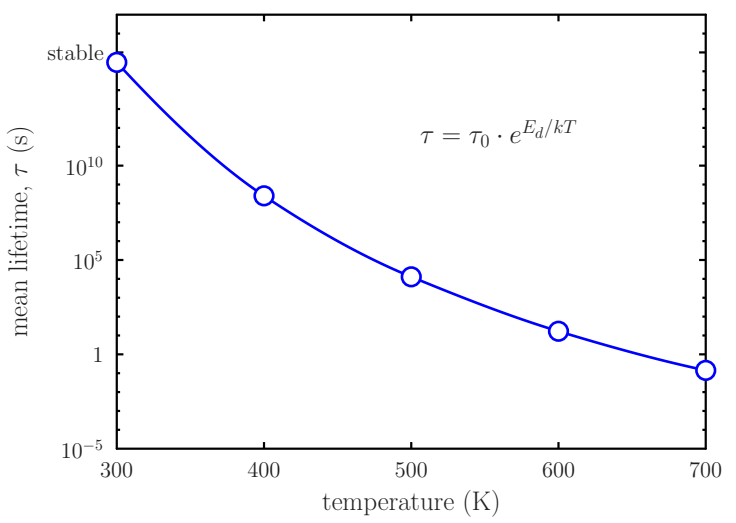

FIG. 6. Mean lifetime of the W di-vacancy as function of temperature obtained with lattice KMC calculations. An Arrhenius fit yields $\tau_{0}=0.093 \pm 0.020$ ps and $E_{d}=1.70 \pm 0.01 \mathrm{eV}$.

present in the real system as a unit (corresponding to the KMC simulations of a single di-vacancy with numerous trials), we can plot the KMC result as a distribution of this population between the different states $(1 \mathrm{NN}, 3 \mathrm{NN}, 4 \mathrm{NN}$ and $5 \mathrm{NN})$ as fractions at each time interval.

In Fig. 3 and Fig. 4 are presented the di-vacancy transition barriers as obtained with NEB-DFT. The heights of the barriers were used as input in the KMC calculations. The

TABLE II. Binding energies of vacancy-C complexes (eV). Different $\mathrm{V}_{2}$-impurity dissociation channels shown for the first $(1 \mathrm{NN})$ and second $(2 \mathrm{NN})$ nearest neighbour $\mathrm{V}_{2}$ configurations.

$\mathrm{AB} \longrightarrow \mathrm{A}+\mathrm{B}$

$\mathrm{V}_{1} \mathrm{C}_{1} \longrightarrow \mathrm{V}_{1}+\mathrm{C}_{1}$

$\mathrm{V}_{2}^{2 \mathrm{NN}} \mathrm{C}_{1} \longrightarrow \begin{cases}\mathrm{V}_{1} \mathrm{C}_{1}+\mathrm{V}_{1} & 1.29 \\ \mathrm{~V}_{2}^{1 \mathrm{NN}}+\mathrm{C}_{1} & 3.33 \\ \mathrm{~V}_{2}^{2 \mathrm{NN}}+\mathrm{C}_{1} & 3.69 \\ \mathrm{~V}_{1} \mathrm{C}_{1}+\mathrm{V}_{1} & 0.04 \\ \mathrm{~V}_{2}^{1 \mathrm{NN}}+\mathrm{C}_{1} & 2.08 \\ \mathrm{~V}_{2}^{2 \mathrm{NN}}+\mathrm{C}_{1} & 2.44 \\ \hline \hline\end{cases}$
$E_{b}$ 


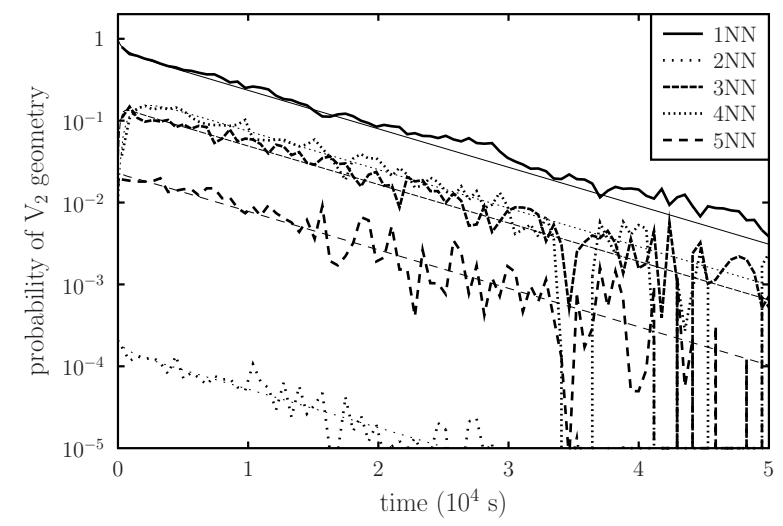

FIG. 7. The occupation time of different $\mathrm{W}$ di-vacancy geometries at $500 \mathrm{~K}$ as obtained with KMC. The most favourable geometry is the $1 \mathrm{NN}$ structure. The $2 \mathrm{NN}$ is the most unstable configuration due to the low transition barriers from $2 \mathrm{NN}$ to $1 \mathrm{NN}$ and $4 \mathrm{NN}$ geometries (see Fig. 3). Straight lines correspond to the results obtained with Master equations (see text for details).

potential barriers for transitions $1 \mathrm{NN} \rightarrow 2 \mathrm{NN} \rightarrow 4 \mathrm{NN} \rightarrow 5 \mathrm{NN}$ were found as $1.68 \mathrm{eV}, 1.55 \mathrm{eV}$ and $1.65 \mathrm{eV}$, respectively. For reverse motion $1 \mathrm{NN} \leftarrow 2 \mathrm{NN} \leftarrow 4 \mathrm{NN} \leftarrow 5 \mathrm{NN}$ the corresponding barriers were obtained as $1.33 \mathrm{eV}, 1.91 \mathrm{eV}$ and $1.55 \mathrm{eV}$. Transitions $1 \mathrm{NN} \rightarrow 3 \mathrm{NN} \rightarrow 4 \mathrm{NN}$ resulted in $1.65 \mathrm{eV}$ and $1.75 \mathrm{eV}$ (reverse motion $1.56 \mathrm{eV}$ and $1.66 \mathrm{eV}$ ) and for $1 \mathrm{NN} \rightarrow 5 \mathrm{NN}$ the barrier was $1.64 \mathrm{eV}$ (reverse motion $1.53 \mathrm{eV}$ ). The di-vacancy was considered to be split, if the random jump took place beyond $5 \mathrm{NN}(1.7 \mathrm{eV})$.

The KMC resulted $\mathrm{W}$ di-vacancy diffusion pre-exponential factor and the effective migration barrier are $D_{0}=0.040 \pm 0.006 \mathrm{~cm}^{2} / \mathrm{s}$ and $E_{m}=1.653 \pm 0.005 \mathrm{eV}$, respectively. These values are comparative with the experimental values for the $\mathrm{W}$ mono-vacancy diffusion (0.04 $\mathrm{cm}^{2} / \mathrm{s}$ and $1.78 \mathrm{eV}$ by Mundy et al. [43]). The obtained theoretical value for mono-vacancy diffusion barrier was $1.7 \mathrm{eV}[27]$.

Fig. 6 presents the calculated di-vacancy mean lifetime, $\tau$, as a function of temperature from RT to $700 \mathrm{~K}$. It can be seen, that once a di-vacancy is formed at RT, it will remain as a stable entity with similar diffusion properties as the mono-vacancy. However, the lifetime of the di-vacancy will rapidly decrease as the temperature increases. At $500 \mathrm{~K}$ the lifetime is $\sim 28 \mathrm{hrs}$ while at $700 \mathrm{~K}$ it is much less than $1 \mathrm{~s}$.

A detailed KMC analysis was done at temperature $500 \mathrm{~K}$ where the di-vacancies are found to be moderately stable. The fractions of all the states within the time span of the 
longest lasting di-vacancy were analysed. This time was divided evenly in 100 bins. Every KMC step of the di-vacancy state was analysed and recorded in a bin of the corresponding time interval when the given transition took place. Every record was weighted by the actual time the transition lasted within the interval. The result of this analysis is presented in Fig. 7. Here, the KMC simulation results are compared to the Master equations written and solved similarly as in Ref. 32. From the Fig. 7 results can be seen, that the 1 NN di-vacancy configuration is the most stable one, since the di-vacancy spends most of the time in this state. The $4 \mathrm{NN}$ and $3 \mathrm{NN}$ configurations are the next most stable di-vacancy structures, whereas the 5NN appears less frequently, which may be an indication of a divacancy residency structure just before it is being dissociated. The most unstable di-vacancy structure in $\mathrm{W}$ is the $2 \mathrm{NN}$ geometry. From Fig. 7 can be seen, that the di-vacancy clearly spends the least time in this geometry, which indicates to an unbound state. Once formed a $2 \mathrm{NN}$ structure, the di-vacancy most probably converts back to a $1 \mathrm{NN}$ geometry. This can be considered as the di-vacancy diffusion mechanism, if the resulted $1 \mathrm{NN}$ is different in lattice orientation than the $1 \mathrm{NN}$ preceding the unstable $2 \mathrm{NN}$ state: the di-vacancy diffusion can be considered propagating via $1 \mathrm{NN} \rightarrow 2 \mathrm{NN} \rightarrow 1 \mathrm{NN}$ mechanism. As a final remark, the clear smooth decreasing tendency of the di-vacancy $\mathrm{NN}$ occupation time evolution profiles is defined by the fluctuations in the lifetimes in the different trials.

The KMC simulations result in the conclusion that the formation of a stable $\mathrm{W}$ divacancy is possible during irradiation events taking place at temperatures $\leq 500 \mathrm{~K}$. However, vacancy clustering via diffusion mechanism to large-sized vacancy clusters is unexpected due to low mono-vacancy-like diffusion pre-exponential factor. As temperatures $>500 \mathrm{~K}$, the di-vacancies become exponentially less stable and the dissociated vacancies may diffuse and form stable vacancy clusters, such as $\mathrm{V}_{4}$. Experimental positron annihilation spectroscopy (PAS) analyses $[14,15]$ in $\mathrm{W}$ at $600-750 \mathrm{~K}$ have shown the $\mathrm{V}_{1}$ related PAS intensity signal to decrease or even vanish, giving rise to a signal component indicating the formation of larger vacancy clusters of $\mathrm{V}_{4}-\mathrm{V}_{10}$.

\section{SUMMARY AND CONCLUSIONS}

To summarise, the properties of small vacancy clusters in bcc $\mathrm{W}$ have been studied computationally. DFT and KMC calculations were used in scrutinizing the stability and 
mobility properties of the di-vacancy. The DFT results indicate the di-vacancy $1 \mathrm{NN}$ and $4 \mathrm{NN}$ configurations being the most stable geometries. It was found, that even though the binding energy $E_{b} \gtrsim 0 \mathrm{eV}$, the di-vacancy can still be found as a stable entity due to the activation energy required for dissociation processes. KMC calculations were done for studying the di-vacancy dissociation dynamics into various lattice directions up to $5 \mathrm{NN}$ at temperatures between RT and $700 \mathrm{~K}$. As a result, an effective dissociation barrier was determined and it was found to be $E_{d}=1.70 \mathrm{eV}$. The di-vacancy diffusion properties were studied accordingly and the effective diffusion parameters found were $D=0.04 \exp (-1.65$ $\mathrm{eV} / k T) \mathrm{cm}^{-2} \mathrm{~s}^{-1}$. The di-vacancy system has a lifetime of $\sim 28$ hours at $500 \mathrm{~K}$. At higher temperatures the system dissociates rapidly and the resulted mono-vacancies are allowed to diffuse freely. The $\mathrm{C}$ impurity was found to make the $2 \mathrm{NN}$ di-vacancy stable. In the presence of impurities, such as $\mathrm{H}$ and $\mathrm{C}$, the $\mathrm{W}$ di-vacancy can get stabilised even at elevated temperatures allowing $\mathrm{V}_{n}$ cluster formation.

\section{ACKNOWLEDGMENTS}

This work has been carried out within the framework of the EUROfusion Consortium and has received funding from the Euratom research and training programme 2014-2018 under grant agreement No 633053. The views and opinions expressed herein do not necessarily reflect those of the European Commission. Grants of computer time from the Center for Scientific Computing (CSC) in Espoo, Finland, and from University of Helsinki, Finland, are gratefully acknowledged.

[1] P. Townsend, J. Kelly, and N. Hartley, Ion implantation, sputtering and their applications. London, New York: Academic Press, 1976.

[2] ITER Physics Basis Editors, et al., "Chapter 1: Overview and summary," Nucl. Fusion., vol. 39 , no. 12 , pp. 2137-2174, 1999.

[3] A. L. et al., "Progress in the ITER Physics Basis Chapter 4: Power and particle control," Nucl. Fusion., vol. 47, no. 6, pp. S203-S263, 2007.

[4] M. Rieth, J. Boutard, S. Dudarev, T. Ahlgren, S. Antusch, N. Baluc, M.-F. Barthe, C. Becquart, L. Ciupinski, J. Correia, C. Domain, J. Fikar, E. Fortuna, C.-C. Fu, E. Gaganidze, 
T. Galán, C. García-Rosales, B. Gludovatz, H. Greuner, K. Heinola, N. Holstein, N. Juslin, F. Koch, W. Krauss, K. Kurzydlowski, J. Linke, C. Linsmeier, N. Luzginova, H. Maier, M. Martínez, J. Missiaen, M. Muhammed, A. Muñoz, M. Muzyk, K. Nordlund, D. NguyenManh, P. Norajitra, J. Opschoor, G. Pintsuk, R. Pippan, G. Ritz, L. Romaner, D. Rupp, R. Schäublin, J. Schlosser, I. Uytdenhouwen, J. van der Laan, L. Veleva, L. Ventelon, S. Wahlberg, F. Willaime, S. Wurster, and M. Yar, "Review on the efda programme on tungsten materials technology and science," J. Nucl. Mater., vol. 417, no. 1-3, pp. 463 - 467, 2011.

[5] N. Taylor, C. Alejaldre, and P. Cortes Fusion Sci. Technol., vol. 64, no. 8-14, p. 111, 2013.

[6] K.-D. Rasch, R. W. Siegel, and H. Schultz, "Quenching and recovery investigations of vacancies in tungsten," Phil. Mag. A, vol. 41, pp. 91-117, 1980.

[7] C. Becquart and C. Domain, "Ab initio calculations about intrinsic point defects and he in w," Nucl. Instr. Meth. Phys. Res. B, vol. 255, no. 1, pp. $23-26,2007$.

[8] M. Muzyk, D. Nguyen-Manh, K. J. Kurzydłowski, N. L. Baluc, and S. L. Dudarev, "Phase stability, point defects, and elastic properties of w-v and w-ta alloys," Phys. Rev. B, vol. 84, p. 104115, 2011.

[9] D. Nguyen-Manh, A. P. Horsfield, and S. L. Dudarev, "Self-interstitial atom defects in bcc transition metals: Group-specific trends," Phys. Rev. B, vol. 73, no. 2, 2006.

[10] D. Kato, H. Iwakiri, and K. Morishita, "Formation of vacancy clusters in tungsten crystals under hydrogen-rich condition," J. Nucl. Mater., vol. 417, no. 1-3, pp. 1115 - 1118, 2011.

[11] J. Y. Park, H.-C. W. Huang, R. W. Siegel, and R. W. Balluffi, "A quantitative study of vacancy defects in quenched tungsten by combined field-ion microscopy and electrical resistometry," Phil. Mag. A, vol. 48, no. 3, pp. 397-419, 1983.

[12] C. Becquart, C. Domain, U. Sarkar, A. DeBacker, and M. Hou, "Microstructural evolution of irradiated tungsten: Ab initio parameterisation of an okmc model," J. Nucl. Mater., vol. 403, no. $1-3$, pp. $75-88,2010$.

[13] L. Ventelon, F. Willaime, C.-C. Fu, M. Heran, and I. Ginoux, "Ab initio investigation of radiation defects in tungsten: Structure of self-interstitials and specificity of di-vacancies compared to other bcc transition metals," J. Nucl. Mater., vol. 425, pp. 16-21, 2012.

[14] H. Eleveld and A. van Veen, "Deuterium interaction with impurities in tungsten studied with tds," J. Nucl. Mater., vol. 191-194, no. Part 1, pp. 433 - 438, 1992. 
[15] H. Eleveld and A. van Veen, "Void growth and thermal desorption of deuterium from voids in tungsten," J. Nucl. Mater., vol. 212-215, pp. 1421-1425, 1994.

[16] G. Kresse and J. Hafner, "Ab initio molecular dynamics for liquid metals," Phys. Rev. B, vol. 47, no. 1, pp. 558-561, 1993.

[17] G. Kresse and J. Hafner, "Ab initio molecular-dynamics simulation of the liquidmetal-amorphous-semiconductor transition in germanium," Phys. Rev. B, vol. 49, no. 20, pp. 14251-14269, 1994.

[18] G. Kresse and J. Furthmüller, "Efficient iterative schemes for ab initio total-energy calculations using a plane-wave basis set," Phys. Rev. B, vol. 54, no. 16, pp. 11169-11186, 1996.

[19] P. E. Blöchl, "Projector augmented-wave method," Phys. Rev. B, vol. 50, no. 24, pp. 1795317979, 1994.

[20] G. Kresse and D. Joubert, "From ultrasoft pseudopotentials to the projector augmented-wave method," Phys. Rev. B, vol. 59, no. 3, pp. 1758-1775, 1999.

[21] J. P. Perdew, K. Burke, and M. Ernzerhof, "Generalized gradient approximation made simple," Phys. Rev. Lett., vol. 77, no. 18, pp. 3865-3868, 1996.

[22] Y. Zhang and W. Yang, "Comment on "generalized gradient approximation made simple"," Phys. Rev. Lett., vol. 80, no. 4, p. 890, 1998.

[23] M. Methfessel and A. T. Paxton, "High-precision sampling for brillouin-zone integration in metals," Phys. Rev. B, vol. 40, no. 6, pp. 3616-3621, 1989.

[24] H. J. Monkhorst and J. D. Pack, "Special points for brillouin-zone integrations," Phys. Rev. $B$, vol. 13 , no. 12 , pp. 5188-5192, 1976.

[25] K. Heinola and T. Ahlgren, "Diffusion of hydrogen in bcc tungsten studied with first principle calculations," J. Appl. Phys., vol. 107, no. 11, p. 113531, 2010.

[26] K. Heinola and T. Ahlgren, "First-principles study of h on the reconstructed w(100) surface," Phys. Rev. B, vol. 81, no. 7, p. 073409, 2010.

[27] T. Ahlgren, K. Heinola, N. Juslin, and A. Kuronen, "Bond-order potential for point and extended defect simulations in tungsten," J. Appl. Phys., vol. 107, no. 3, p. 033516, 2010.

[28] G. Mills, H. Jonsson, and G. K. Schenter, "Reversible work transition state theory: application to dissociative adsorption of hydrogen," Surf. Sci., vol. 324, no. 2-3, pp. 305-337, 1994.

[29] H. Jonsson, G. Mills, and K. W. Jacobsen, Classical and Quantum Dynamics in Condensed Phase Simulations. World Scientific, 1998. 
[30] N. Soneda and T. D. de la Rubia, "physics of condensed matter structure defects and mechanical properties," Phil. Mag. A, vol. 78, p. 995, 1998.

[31] M. J. Caturla, N. Soneda, E. Alonso, B. D. Wirth, T. D. de la Rubia, and J. M. Perlado J. Nucl. Mater., vol. 276, p. 13, 2000.

[32] F. Djurabekova, L. Malerba, R. C. Pasianot, and P. Olsson Phil. Mag., vol. 90, p. 2585, 2010.

[33] F. G. Djurabekova, R. Domingos, G. Cerchiara, N. Castin, E. Vincent, and L. Malerba Nucl. Instr. Meth. Phys. Res. B, vol. 255, p. 8, 2007.

[34] F. G. Djurabekova, L. Malerba, C. Domain, and C. S. Becquart Nucl. Instr. Meth. Phys. Res. $B$, vol. 255, p. 45, 2007.

[35] C. Domain, C. S. Becquart, and J.-C. V. Duysen Mater. Res. Soc. Symp. Proc., vol. 540, p. $643,1999$.

[36] A. B. Bortz, M. H. Kalos, and J. L. Lebowitz J. Comp. Phys., vol. 17, p. 10, 1975.

[37] K. Heinola, T. Ahlgren, K. Nordlund, and J. Keinonen, "Hydrogen interaction with point defects in tungsten," Phys. Rev. B, vol. 82, no. 9, p. 094102, 2010.

[38] C. Becquart and C. Domain, "A density functional theory assessment of the clustering behaviour of he and h in tungsten," J. Nucl. Mater., vol. 386-388, pp. 109 - 111, 2009.

[39] Y. Oda, A. M. Ito, A. Takayama, and H. Nakamura, "First-principles study on migrtion of vacancy in tungsten," Plasma Fusion Res., vol. 9, p. 3401117, 2014.

[40] F. Willaime, A. Satta, M. Nastar, and O. L. Bacq, "Electronic structure calculations of vacancy parameters in transition metals: Impact on the bcc self-diffusion anomaly," Int. J. Quantum Chem., vol. 77, pp. 927-939, 2000.

[41] D. Nguyen-Manh and S. L. Dudarev, "Trapping of he clusters by inert-gas impurities in tungsten: First-principles predictions and experimental validation," Nucl. Instr. Meth. Phys. Res. B, vol. 352, pp. 86-91, 2015.

[42] F. Djurabekova, L. Malerba, R. Pasianot, P. Olsson, and K. Nordlund, "Kinetics versus thermodynamics in materials modeling: The case of the di-vacancy in iron," Phil. Mag., vol. 90, no. 19, pp. 2585-2595, 2010.

[43] J. N. Mundy, S. J. Rothman, N. Q. Lam, H. A. Hoff, and L. J. Nowicki, "Self-diffusion in tungsten," Phys. Rev. B, vol. 18, no. 12, pp. 6566-6575, 1978. 